\title{
BMJ Open Biopsychosocial factors associated with non-recovery after a minor transport- related injury: protocol for a systematic review
}

\author{
Stella Samoborec, ${ }^{1}$ Rasa Ruseckaite, ${ }^{1}$ Lorena Romero, ${ }^{2}$ Sue M Evans ${ }^{1,3}$
}

To cite: Samoborec $S$, Ruseckaite R, Romero L, et al. Biopsychosocial factors associated with non-recovery after a minor transportrelated injury: protocol for a systematic review. BMJ Open 2017;7:e016314. doi:10.1136/ bmjopen-2017-016314

- Prepublication history and additional material for this paper are available online. To view please visit the journal (http:// dx.doi.org/10.1136/bmjopen2017-016314).

Received 7 February 2017

Revised 28 July 2017

Accepted 31 July 2017
CrossMark

${ }^{1}$ Department of Epidemiology and Preventive Medicine, School of Public Health and Preventive Medicine, Monash University, Melbourne, Victoria, Australia ${ }^{2}$ The lan Potter Library, Alfred Hospital, Melbourne, Victoria, Australia

${ }^{3}$ Clinical Registry Unit, Monash University, Melbourne, Victoria, Australia

Correspondence to

Stella Samoborec;

stella.samoborec@monash.edu

\section{ABSTRACT}

Introduction Globally, road transport accidents contribute substantially to the number of deaths and also to the burden of disability. Up to 50 million people suffer a transport-related non-fatal injury each year, which often leads to long-term disability. It has been shown that substantial number of people with minor injuries struggle to recover and the reasons are still not well explored. Despite the high prevalence, little is known about the factors hindering recovery following minor traffic-related injuries. The aim of this paper is to present a protocol for the systematic review aiming to understand biopsychosocial factors related to non-recovery and identify current gaps in the literature.

Methods and analysis The review will be conducted in compliance with the Preferred Reporting Items for Systematic Reviews and Meta-Analysis Protocol guidelines. A search of the electronic databases, MEDLINE, EMBASE, Cochrane Central Register of Controlled trials, will be undertaken, in addition to Google Scholar and grey literature to identify studies in period from 2006 to 2016 . Quantitative and qualitative research articles describing and identifying biopsychosocial factors associated with non-recovery and health outcomes such as pain, disability, functional recovery, health-related quality of life, posttraumatic stress disorder, depression, anxiety and return to work will be included. A conceptual framework developed to identify biopsychosocial factors will be applied to assure defined criterion. At present, there is little anticipation for meta-analyses due to the heterogeneity of factors and outcomes assessed. Therefore, a narrative synthesis based on study findings will be conducted.

Ethics and dissemination Ethical approval is not required as primary data will not be collected. Review results will be published as a part of thesis, peer-reviewed journal and conferences.

Trialregistration number PROSPEROregistration number: CRD42016052276.

\section{INTRODUCTION}

Worldwide, road transport accidents contribute substantially to the number of deaths and also to the burden of disability. WHO estimates that by 2020 road accidents will be the third leading cause of disability. ${ }^{1}$

\section{Strengths and limitations of this study}

- This will be the first systematic review evaluating biopsychosocial factors associated with nonrecovery across the different types of minor transport-related injuries.

- The review has distinct inclusion criteria and clearly outlines how the items will be selected and abstracted.

- The review aims to offer highest level of evidence on factors deterring recovery after minor traffic-related injuries.

- However, due to the variety of factors and relevant outcomes, comparison of the outcomes may not be possible.

- The potential issue of heterogeneity across the studies may affect the study results.

According to WHO data, deaths from road traffic injuries account for around $25 \%$ of all deaths from injury. ${ }^{2}$

Minor injuries are the most recurrently reported injuries following a transport-related accident. ${ }^{3}$ While the number will fluctuate between countries, the literature suggests that the total incidence of minor injuries (musculoskeletal and soft tissue) has increased in the last 30 years. ${ }^{4}$ Whiplash and whiplash-associated disorder (WAD) are the most frequently reported minor injuries following a transport accident. ${ }^{3}{ }^{5-10}$ Other minor injuries include contusions, skin abrasions, lacerations, sprains and strains, as defined by Minor Injury Guidelines. The guideline defines a Minor injury as follows: 'minor injury means a sprain, strain, WAD, contusion, abrasion, laceration or subluxation and any clinically associated sequelae. This term is to be interpreted to apply where a person sustains any one or more of these injuries'. ${ }^{11}$ Despite a substantial amount of WAD epidemiology and treatment research, understanding factors that hinder and 
obstruct recovery for WAD and other minor injuries is scant. ${ }^{5}$

The complexity and heterogeneity of the profile, of those suffering minor traffic-related injuries are reasons to explain why many people do not recover as expected. ${ }^{12}$ It has been estimated that approximately half of the patients with minor injuries may never completely recover ${ }^{13}$ and large proportion of people with WAD would suffer psychological distress for at least 3 years postaccident. ${ }^{14}$

In Victoria, while preventive methods have been directed to patients with major injuries, there are no preventive recommendations and rehabilitative guidelines for patients with minor injuries. Yet, it is believed that there is much to be achieved by understanding factors and interventions aimed at reducing long-term disability and improving recovery for those who have sustained minor injuries. ${ }^{15}$ It is also important to note that there are various complexities in treating and managing patients with minor injuries. Although it is expected that not everyone who sustains a minor injury will develop persistent symptoms, cautious consideration is required to understand and identify in a timely manner those patients with minor injuries who are at high risk of protracted recovery.

\section{Minor transport-related musculoskeletal injuries}

The severity of injuries between different groups and patients are compared according to different scales. Numerous injury severity scales exist in practice and in the literature. However, the assessment of motor vehicle injuries relies mainly on the Abbreviated Injury Scale (AIS). ${ }^{16}$ AIS is the first broadly implemented injury severity scale used in practice and is primarily an anatomical measure of injury severity. It classifies severity on the basis of the region of the body injured and the degree of the injury in that particular body region. For example, an AIS score of 1 interprets a minor injury, while an AIS score of 6 is considered as a non-survivable injury. It is important to note that the scores from 1 to 6 do not reflect an interval scale, and comparable AIS scores may not be similar across different body regions. In summary, a higher severity score indicates a gradually more severe injury. $^{17}$

The most common types or minor transport-related injuries are musculoskeletal and/or soft tissue injuries. ${ }^{18}$ Musculoskeletal injuries refer to those which affect muscles, bones, joints, tendons, ligaments, cartilage and spinal discs. Soft tissue injuries can arise in any soft tissue in the body. If they occur in the skin, they are known as contusions, in the muscle they are identified as strains, and in the tendons and ligaments, they are recognised as sprains. ${ }^{19}$ While some of these injuries are benign and do not require complex treatments, others may lead to chronic and persistent challenges. ${ }^{20}{ }^{21}$ The cause of protracted symptoms are thought to be complex and multifactorial. According to the literature, these conditions are often shown to be painful and require medical intervention. Minor injuries are usually treated in primary healthcare. However, some require specialist intervention, treatment and, in some cases, hospitalisation. ${ }^{22}$ It is to note that there is no current evidence of types and number of medical treatments which would be most beneficial for patients with minor traffic-related injuries.

\section{Rationale and objectives}

There is still paucity of research into predictors and determinants of recovery following minor injuries. In clinical practice, there remains a lack of recognition that patients with minor injury may have a slow recovery and longterm adverse biopsychosocial consequences. ${ }^{3}$ Previous research demonstrates differences in patient's recovery outcomes and identifies a number of factors leading to long-term disability and poor health outcomes. ${ }^{23-27}$ However, the results are not consisted and generalisable to larger population. It is evident that more research is needed to understand and investigate whether early identification of the most predictive factors could reduce chronicity and long-term disability. It is also believed that the quality of management of the most common types of minor injuries should be improved. ${ }^{28}$ In conclusion, these patients should be identified as early as possible in their injury trajectory so that active support and management can be provided.

The objectives of the proposed systematic review are to identify and assess biopsychosocial factors and relevant predictors of non-recovery and determine the benefits of using biopsychosocial model (BPS) or approach on identifying health outcomes after minor transport-related injury.

\section{METHODS AND ANALYSES}

A detailed description on population, intervention, comparison and outcome of the systematic review is outlined in table 1 and described below:

\section{Inclusion criteria}

Articles will be included if they are:

- Investigating patients sustained minor transport-related injury;

- Assessing biological, psychological and social factors as defined by BPS model of health ${ }^{29}$;

- Using BPS model of health as a core model or approach for identifying health outcomes;

- Published in English language;

- Published in the last decade (from January 2006 to December 2016).

\section{Exclusion criteria}

Articles will be excluded if they were:

- Published in a language other than English;

- Published prior to January 2006 or after December 2016;

- Describing work-related injury;

- Not using validated tools to measure recovery outcomes; 
Table 1 Description of the population, intervention, comparison and outcome (PICO) of the systematic review

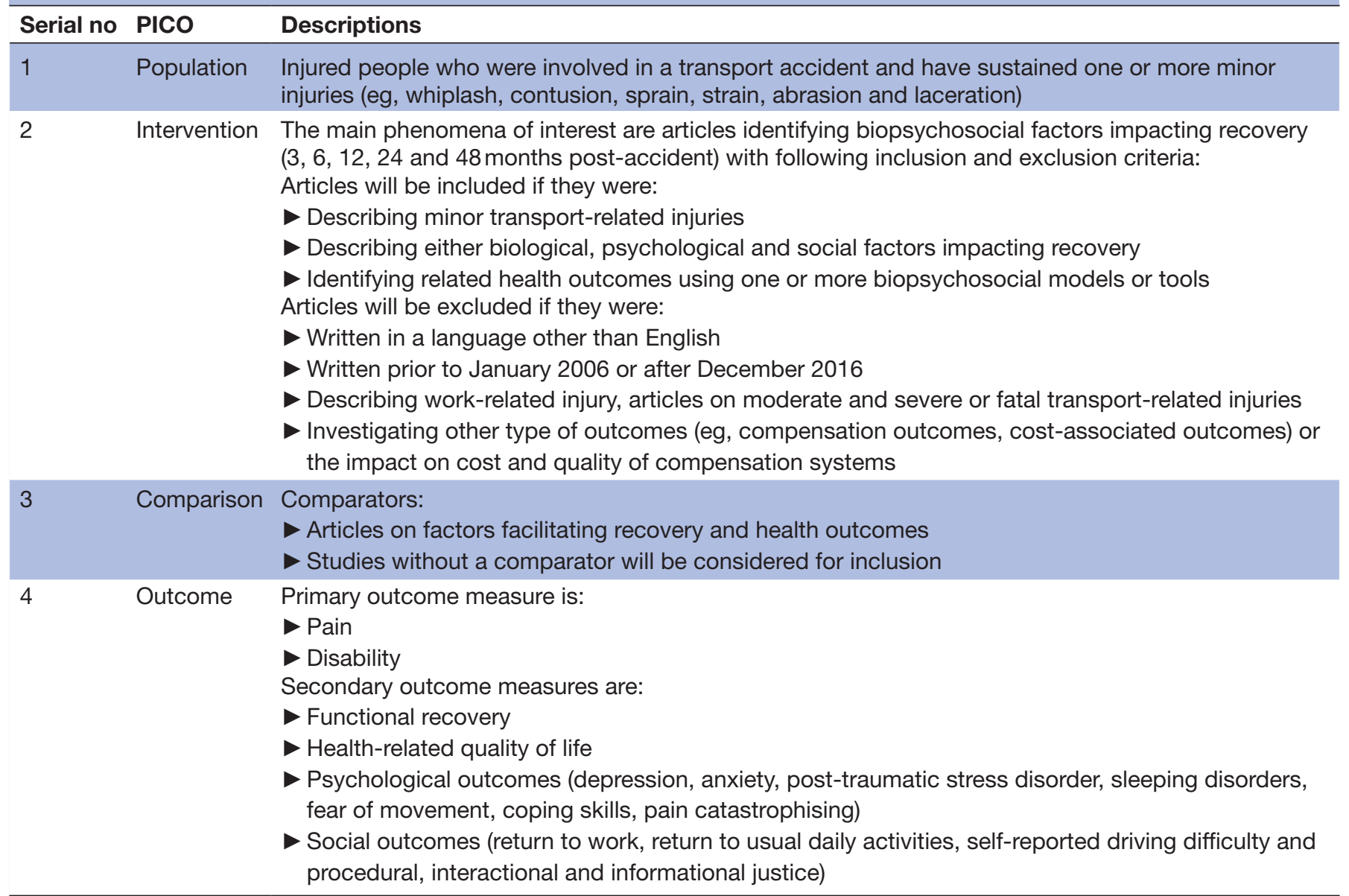

- Involving children and describing paediatric injuries;

- Describing moderate and severe or fatal transport-related injuries (based on AIS scores of 2-6);

- Investigating other type of outcomes (eg, compensation outcomes such as cost, time to claim closure, impact on cost and quality of compensation systems or services);

- Unpublished manuscripts, dissertations, books and book chapters, conference proceedings, meeting abstracts, and guideline statements will be excluded.

\section{Study design}

Quantitative (eg, cohort, longitudinal, case studies, prospective and retrospective) and qualitative studies (eg, ethnography, phenomenological, grounded theory and case report) exploring biopsychosocial factors impacting recovery and related health outcomes in patients with minor transport-related injury will be included. Mixed methods research articles will also be included in the review.

\section{Comparator(s)/control}

Comparators such as positive factors and factors enabling recovery after minor transport-related accident will be considered for inclusion.

\section{Context}

Studies conducted in the clinical environments such as acute care (emergency departments) and subacute care (primary healthcare, pain clinics, rehabilitation centres) will be included. Settings such as insurance databases and registries will also be included.

\section{Outcome measure/outcome of interest}

The following outcomes will be investigated:

- Functional recovery (eg, return to pre-accident level of functionality or independence or usual activities)

- Disability (eg, temporary, long-term, permanent)

- Pain intensity (eg, low, moderate, severe)

- Health-related quality of life (eg, poor, good)

- Psychological outcomes (eg, depression, fear, sleep disorder, anxiety, post-traumatic stress disorder)

- Social outcomes (eg, socioeconomics, return to work, family and community support, quality of healthcare).

\section{Search methods}

The database records and details of how the search was undertaken will be maintained at each stage of the review process. A senior medical librarian (LR) will assist in the final draft of the search strategy. 
The suggested review will search the following electronic databases: MEDLINE, EMBASE, Cochrane Central Register of Controlled Trials and the Google Scholar. If relevant, grey literature such as government reports may also be included. The search strategy will be developed in Medline and then adopted to the other databases. It will include the subject headings specific to each database and a free text word specific to review inclusion criteria. The complete search strategy can be seen in online supplementary appendix 1. Databases containing the results of the searches will be created using EndNote X7.

\section{Study screening and selection}

A three-phase screening process will be applied. In phase one, an experienced medical librarian (LR) and a researcher (SS) will conduct the initial search. In a second phase, two researches (SS, SME) will independently screen the tittles and abstracts of all articles identified in the search strategy to determine eligibility and classify studies as relevant, possibly relevant and irrelevant. During the last phase, the researches (SS, RR) will independently review the full text to make a final determination of eligibility. Any disagreements that arise between the reviewers will be resolved through a discussion and consensus. The Preferred Reporting Items for Systematic Reviews and Meta-Analysis Protocol methodology, checklist and standard search strategy using predefined inclusion and exclusion criteria and structured data abstraction tools will be used.

\section{Data extraction}

Data from the relevant articles will be assessed based on the Cochrane data abstraction form. ${ }^{30}$ The data will be extracted by two reviewers (SS, RR) and any inconsistencies arising will be identified and resolved through discussion with a third reviewer. Evidence will be synthesised based on the following information:

- study period (start and end date)

- study population (number of participants)

- type of study (quantitative or qualitative)

- injury studied (type and severity of injury)

- outcome/s of interest

- tools used to identify outcomes

- type of factors (biological, psychological and social)

- effect and directions of biopsychosocial factors on outcome/s (prediction and impact)

- limitations of study

- key findings and recommendations.

\section{Data management}

The relevant review documentation and search results will be uploaded and saved in faculty-allocated network storage (S-drive) located in Monash University and will be backed up on faculty-allocated network storage. The data will be accessed only by the reviewers.

\section{Study quality and assessing risk of bias}

A critical appraisal for quantitative studies will be made using the Scottish Intercollegiate Guideline Network (SIGN) tool to assess risk of bias for individual quantitative studies included in the review. ${ }^{31}$ SIGN provides checklists to assess the quality of systematic reviews and meta-analyses, randomised control trials, cohort studies, case-control studies, diagnostic studies and economic studies. This criteria will assist with the evaluation of the impact of detection, selection, performance, information bias and confounding on study results. Two review authors (SS, RR) will independently appraise the methodology of the included studies and categorise the study as being of high $(++)$, acceptable $(+)$ or unacceptable (0) quality. Qualitative studies will be assessed based on the Cochrane guidance for inclusion of qualitative research in systematic reviews. ${ }^{32}$ Core elements of credibility, transferability, dependability and confirmability will be assessed and reported. The Standards for Reporting Qualitative Research ${ }^{33}$ tool covers all the recommended criteria for assessing risk of bias in qualitative studies and will be used for critically appraising methodology of qualitative studies. Any discrepancies arising will be discussed between the reviewers.

\section{ANALYSIS}

\section{Descriptive analysis}

The conceptual framework has been developed to identify biopsychosocial factors impacting recovery and relevant health outcomes (figure 1). The Cochrane data abstraction criteria ${ }^{30}$ will be used to synthesise the results of the included studies.

\section{Statistical analysis}

Unavoidably, number of different studies brought together will differ and high variability is expected for the proposed review. It is anticipated that there will be limited capacity to undertake a meta-analysis because of the range and the heterogeneity of the factors, outcomes and profile of those who have sustained a minor transport-related injury. However, careful consideration will be undertaken involving a consultation with a systematic review experts based on the attributes of the included

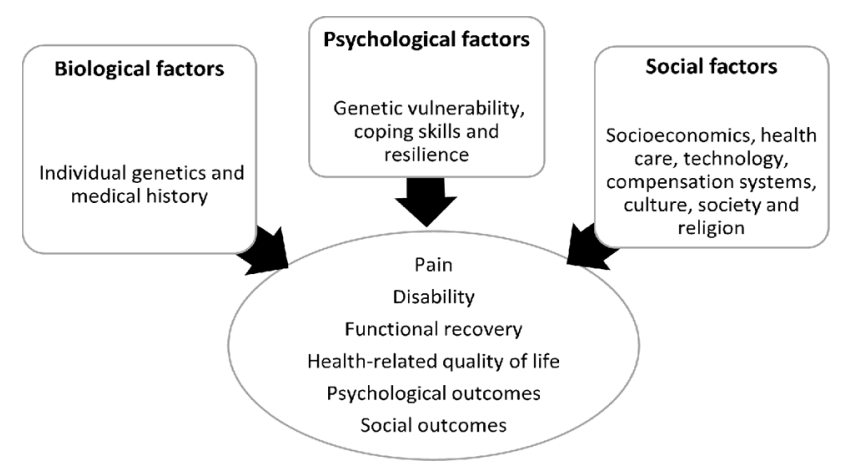

Figure 1 Conceptual framework for identifying factors impacting recovery after traffic-related accident. 
studies. If a decision is made to conduct a meta-analysis, reviewers will consider recommendations on selecting an appropriate method for dealing with heterogeneity in meta-analysis outlined by Schroll et al. ${ }^{34}$ We will likely consider random-effect meta-analysis as it is highly unlikely that all studies will be functionally equal. If we determined that heterogeneity is too large and decide not to pursue meta-analysis, we will present descriptive analyses of the included studies.

\section{DISCUSSION}

The proposed review aims to improve understanding of non-recovery after minor injuries and its associated factors. It intends to assess the best available evidence of the biopsychosocial factors hindering recovery following a minor transport-related accident. The review main aim is to provide a detailed description of a range of biological, psychological and social factors and explain why some people with minor injuries do not recover as expected.

The results of this study should form the basis to better understand recovery after minor injury and inform health policy and clinical management about current evidence in the literature.

However, it is to note that there will be challenges in the review process and also in interpreting findings. First, the evaluation of the primary outcomes will depend on the intervention and tools used to identify these outcomes. Second, as some minor injuries do not require hospitalisation, less physical proof will be available for this group. Third, data on social outcomes may not be representative as it may not be reported in a sufficient number of studies.

In conclusion, the proposed systematic review will aim to identify gaps in the current knowledge and provide a detailed summary of factors deterring recovery at different time points after traffic-related injury based on the BPS model of health.

Acknowledgements We thank Capital Markets Cooperative Research Centre and Transport Accident Commission for financial support.

Contributors SS, SME and RR have contributed in developing the idea and methodology for systematic review. SS registered the protocol with PROSPERO and drafted the first manuscript which was reviewed by all the authors. The constructive feedback was given from SME and RR and encompassed in the final version. The final version was critically revised by all the authors and finalised by SS. All authors read and approved the final manuscript.

Funding SS (Monash ID 26381494) has received Capital Markets Cooperative Research Centre and Transport Accident Commission living allowance scholarship for conducting this study.

Competing interests None declared.

Provenance and peer review Not commissioned; externally peer reviewed.

Open Access This is an Open Access article distributed in accordance with the Creative Commons Attribution Non Commercial (CC BY-NC 4.0) license, which permits others to distribute, remix, adapt, build upon this work non-commercially, and license their derivative works on different terms, provided the original work is properly cited and the use is non-commercial. See: http://creativecommons.org/ licenses/by-nc/4.0/

(C) Article author(s) (or their employer(s) unless otherwise stated in the text of the article) 2017. All rights reserved. No commercial use is permitted unless otherwise expressly granted.

\section{REFERENCES}

1. World Health Organisation. World report on road traffic injury prevention. Washington D.C: World Health Organisation, 2004.

2. World Health Organisation. World report on road traffic injury prevention. Geneva, Switzerland, 2004.

3. Casey PP, Feyer AM, Cameron ID. Identifying predictors of early nonrecovery in a compensation setting: The Whiplash Outcome Study. Injury 2011;42:25-32.

4. Peden M, Sminkey L. World Health Organization dedicates World Health Day to road safety. Inj Prev 2004;10:67.

5. Casey PP, Feyer AM, Cameron ID. Course of recovery for whiplash associated disorders in a compensation setting. Injury 2015;46:2118-29.

6. Adams H, Ellis T, Stanish WD, et al. Psychosocial factors related to return to work following rehabilitation of whiplash injuries. J Occup Rehabil 2007;17:305-15.

7. Atherton $\mathrm{K}$, Wiles NJ, Lecky FE, et al. Predictors of persistent neck pain after whiplash injury. Emerg Med J 2006;23:195-201.

8. Buitenhuis $\mathrm{J}$, de Jong PJ, Jaspers JP, et al. Work disability after whiplash: a prospective cohort study. Spine 2009;34:262-7.

9. Crutebo S, Nilsson C, Skillgate E, et al. The course of symptoms for whiplash-associated disorders in Sweden: 6-month followup study. $J$ Rheumatol 2010;37:1527-33.

10. Holm LW, Carroll LJ, Cassidy JD, et al. Expectations for recovery important in the prognosis of whiplash injuries. PLoS Med 2008;5:e105.

11. Côté $\mathrm{P}$, Shearer $\mathrm{H}$, Ameis $\mathrm{A}$, et al. Enabling recovery from common traffic injuries: A focus on the injured person. UOIT-CMCC Centre for the Study of Disability Prevention and Rehabilitation, 2015.

12. Gopinath B, Jagnoor J, Harris IA, et al. Comparison of health outcomes between hospitalised and non-hospitalised persons with minor injuries sustained in a road traffic crash in Australia: a prospective cohort study. BMJ Open 2015;5:e009303.

13. Berecki-Gisolf $\mathrm{J}$, Collie A, McClure R. Reduction in health service use for whiplash injury after motor vehicle accidents in 2000-2009: results from a defined population. J Rehabil Med 2013;45:1034-41.

14. Craig A, Tran Y, Guest R, et al. Psychological impact of injuries sustained in motor vehicle crashes: systematic review and metaanalysis. BMJ Open 2016;6:e011993.

15. Sullivan MJ, Stanish W, Sullivan ME, et al. Differential predictors of pain and disability in patients with whiplash injuries. Pain Res Manag 2002;7:68-74.

16. Lopes MC, Whitaker IY. [Measuring trauma severity using the 1998 and 2005 revisions of the abbreviated injury scale]. Rev Esc Enferm USP 2014;48:641-8.

17. Lesko MM, Woodford M, White L, et al. Using Abbreviated Injury Scale (AIS) codes to classify Computed Tomography (CT) features in the Marshall System. BMC Med Res Methodol 2010;10:72.

18. Rosenbloom BN, Khan S, McCartney C, et al. Systematic review of persistent pain and psychological outcomes following traumatic musculoskeletal injury. J Pain Res 2013;6:39.

19. Svestková O. International classification of functioning, disability and health of World Health Organization (ICF). Prague Med Rep 2008;109:268-74.

20. Gopinath B, Jagnoor J, Nicholas M, et al. Presence and predictors of persistent pain among persons who sustained an injury in a road traffic crash. Eur J Pain 2015;19:1111-8.

21. Gopinath B, Harris IA, Nicholas M, et al. A comparison of health outcomes in older versus younger adults following a road traffic crash injury: a cohort study. PLoS One 2015;10:e0122732.

22. Berecki-Gisolf $\mathrm{J}$, Collie A, McClure R. Work disability after road traffic injury in a mixed population with and without hospitalisation. Accid Anal Prev 2013;51:129-34.

23. Derrett S, Samaranayaka A, Wilson S, et al. Prevalence and predictors of sub-acute phase disability after injury among hospitalised and non-hospitalised groups: a longitudinal cohort study. PLoS One 2012;7:e44909.

24. Giummarra MJ, Ioannou L, Ponsford J, et al. Chronic pain following motor vehicle collision: a systematic review of outcomes associated with seeking or receiving compensation. Clin J Pain 2016;32:817-27.

25. Harris IA, Young JM, Jalaludin BB, et al. The effect of compensation on general health in patients sustaining fractures in motor vehicle trauma. J Orthop Trauma 2008;22:216-20.

26. Murgatroyd DF, Casey PP, Cameron ID, et al. The effect of financial compensation on health outcomes following musculoskeletal injury: systematic review. PLoS One 2015;10:e0117597.

27. Ozegovic D, Carroll LJ, Cassidy JD. What influences positive return to work expectation? Examining associated factors in a population-based cohort of whiplash-associated disorders. Spine 2010;35:E708-13. 
28. Collie A, Gabbe B, Fitzharris M. Evaluation of a complex, populationbased injury claims management intervention for improving injury outcomes: study protocol. BMJ Open 2015;5:e006900.

29. Engel GL. The need for a new medical model: a challenge for biomedicine. Science 1977;196:129-36.

30. Cochrane Effective Practice and Organisation of Care. http://epoc. cochrane.org/epoc-specific-resources-review-auth (accessed Dec 2016).

31. SIGN. Scottish intercollegiate guidelines network for cohort and case control studies. http://www.sign.ac.uk/checklists-and-notes.html (accessed Feb 2017).
32. Hannes K. et alSupplementary Guidance for Inclusion of Qualitative Research in Cochrane Systematic Reviews of Interventions. In: Noyes JBA, Hannes K, Harden A, . eds. Critical appraisal of qualitative research: Cochrane Collaboration Qualitative Methods Group. 2011.

33. O'Brien BC, Harris IB, Beckman TJ, et al. Standards for reporting qualitative research: a synthesis of recommendations. Acad Med 2014;89:1245-51.

34. Schroll JB, Moustgaard R, Gøtzsche PC. Dealing with substantial heterogeneity in Cochrane reviews. Cross-sectional study. BMC Med Res Methodol 2011;11:22. 